\title{
Extraskeletal and skeletal Ewing sarcoma family of tumours in adults: a clinico radiological review of 50
}

\section{cases}

\begin{abstract}
This review describes the imaging characteristics of Ewing sarcoma family of tumours (ESFT) exclusively in an adult population. The magnetic resonance imaging (MRI) and computed tomography (CT) appearances in 50 adult patients with ESFT is described. The imaging characteristics in this largest case series of adult ESFT reported so far, were similar to those reported in the paediatric ESFT. Our incidence of extra skeletal ESFT of $18 \%$ is the highest reported so far in the adult population and is higher than the reported incidence of $(8 \%)$ in the paediatric and adolescent population. MRI was found to be superior to CT scan in identifying tumour involvement of the bone marrow and in delineating the soft tissue involvement due to its multi-planar imaging capability.
\end{abstract}

Keywords: ewing sarcoma, magnetic resonance studies, computed tomography, magnetic resonance comparative studies
Volume 3 Issue I - 2017

\author{
Prangama Chatterjee, ' Ashish Singh, ${ }^{2}$ Vinay \\ Kuruvilla ${ }^{2}$ \\ 'Department of Radiodiagnosis and Imaging, Christian Medical \\ College, India \\ ${ }^{2}$ Department of Medical Oncology, Christian Medical College, \\ India
}

Correspondence: Parangama Chatterjee, Department of Radiodiagnosis and Imaging, Christian Medical College, Vellore-632004,Tamil Nadu, India, Tel 9894722569, 04I 622830I2, Email parangamadr@gmail.com

Received: March 31, 2017| Published: June 08, 2017
Abbreviations: ESFT, ewing sarcoma family of tumours; MRI, magnetic resonance imaging; CT, computed tomography; ES, ewing sarcoma; PNET, peripheral neuroectodermal tumor; EFT, ewing sarcoma family of tumors; STIR, sequences with inversion recovery

\section{Introduction}

Ewing sarcoma (ES) and primitive peripheral neuroectodermal tumor (PNET) were originally described as distinct clinicopathologic entities by James Ewing in 1921 and Stout in 1918 respectively. At present, it is known that these entities comprise the same spectrum of tumours known as the Ewing sarcoma family of tumors (EFT), ${ }^{1}$ which also includes malignant round-cell tumor of the thoracopulmonary region (Askin's tumor), and atypical ES. The EFT can develop in nearly any bone or soft tissue, but the commonest site is in a flat or long bone. The typical patient presents with localized pain and swelling. ${ }^{2}$ Although metastatic disease is found in lesser than 25percent at presentation, ${ }^{3}$ subclinical metastatic disease is assumed to be present in almost all patients because of the 80 to 90percent relapse rate in patients undergoing local therapy alone. ${ }^{4}$ The diagnostic work-up is usually initiated with a plain radiograph of the affected region. Compared to plain radiographs, a CT scan better delineates the extent of cortical destruction and soft tissue involvement. CT imaging features include a heterogeneous appearing mass with poorly defined irregular margins, displacing surrounding tissues with mixed internal attenuation, similar to muscle with necrosis and/or calcification. However, definition of tumor size, local intraosseous and extraosseous extent, and the relationship of the tumor to fascial planes, vessels, nerves, and organs are best achieved by magnetic resonance imaging (MRI)

On MRI, these tumours usually have a pattern of heterogeneous low and high signal on $T_{1}$ and $T_{2}$ weighted images respectively, due to occasional haemorrhage and frequent extensive necrosis. Imaging of the entire involved bone is necessary to exclude the presence of skip lesions. In this study, we analysed our experience of 50 patients with ES, who were presurgically investigated using CT and/or MR, and describe the anatomical distribution and imaging features of this tumour.

\section{Materials and methods}

This was a descriptive retrospective study carried out according to our institutional guidelines. For patients with multiple imaging the initial staging CT/MR was evaluated for analysis. Computed tomography was carried out after an overnight fasting and was carried out by using two CT scanners Philips Brilliance (Philips Medical Systems, Iselin, NJ, USA; six-slice multi detector computed tomography) and Siemens Emotion (Siemens Medical Systems, single slice Helical CT scanner). A total of $100 \mathrm{~mL}$ of non-ionic contrast material was injected i.v. at a rate of $3 \mathrm{~mL} / \mathrm{sec}$ by using a power injector. Two-phase scans were obtained 30sec (early/arterial phase) and70 sec (venous/delay phase) after the start of i.v.injection. All scans were obtained in a single breath-hold from the diaphragmatic dome to the pubis symphysis. The parameters were $120 \mathrm{kVp}$ and $280 \mathrm{mAs}$, or $130 \mathrm{kVp}$ and $140 \mathrm{mAs}$, with a pitch of 1.5 .

\section{MR imaging examination}

Coils cardiac: Phased array external coils.

Sequence protocols: MR imaging was performed at our institution at 1.5T (Siemens Medical Systems, Best, Netherlands) with $18.0 \mathrm{mT} / \mathrm{m}$, a rise time of $0.16 \mathrm{~m} \mathrm{sec}$, and a slew rate of $200 \mathrm{mT} / \mathrm{m} / \mathrm{sec}$. Subjects were positioned supine and in the head first position.

Sequences used were precontrast T1-weighted two-dimensional turbo spin echo $(656 / 10 \mathrm{msec}$; echo train length, five; section thickness, $8 \mathrm{~mm}$; intersection gap, $0.8 \mathrm{~mm}$; number of signals acquired, four; matrix, 166x256; field of view, $25 \mathrm{~cm}$; and T2-weighted twodimensional turbo spin echo high resolution images $(3,427 / 150$; echo 
train length, 25; section thickness, $4 \mathrm{~mm}$; intersection gap, $0.8 \mathrm{~mm}$; number of signals acquired, eight; matrix, 175x256; field of view, $20 \mathrm{~cm}$; voxel size, $2.43 \mathrm{~mm}^{3}$ ).

T1-weighted sequence was performed in the transverse plane, and these served as a reference for accurate planning of the sagittal T2weighted turbo spin-echo sequence. Sagittal T2-weighted turbo spinecho images were then used for accurate planning of the transverse and coronal T2-weighted turbo spin-echo sequences. The axial and coronal T2-weighted TSE sequence was angled perpendicular to the long axis of the tumour. Gadolinium was not administered.

The findings were retrospectively evaluated by a radiologist. All images were reviewed on a 2000x2000 Picture Archiving and Communication system monitor. Between January 2003 and December 2009, 50 consecutive patients were evaluated, of whom 38 patients underwent surgery in our institute. In the patients who did not undergo surgery, diagnosis was confirmed by biopsy and immunohistochemistry. Ewing's PCR was done in those patients in whom the diagnosis was in doubt for confirmation.

\section{Results}

For the 50 patients evaluated, data was obtained from $26 \mathrm{CT}$ scans and $30 \mathrm{MRI}$ scans that were performed on these patients. Demographic distribution included 40 men and 10 women; age range was from 19 to 40 years. The mean age of the patients at the time of diagnosis was 23.2 years. The location of the tumours was predominantly in the chest wall, limbs and extra skeletal sites, as shown in Table 1.9 patients had extraskeletal tumours. The mean diameter of the primary tumour was $8.2 \mathrm{~cm}$ (range: 8 to $20 \mathrm{~cm}$ ). The tumour margin was well preserved (i.e. smooth or lobular) in 34 patients. Central fluid attenuation was present in 20 tumours, and calcification in eight patients. One patient had skip lesions, 2 patients had hemorrhagic lesions. Almost all lesions were of low signal on T1 images and high signal on T2 images. Metastasis was present in eight patients at presentation. Among all patients, 16 showed evidence of adjacent organ invasion, and 4 showed adjacent neuro vascular invasion. Some rare interesting sites such as ovary, prostate, rectum, scrotum, brain and pancreatic tail were encountered (Figures 1-4). The other sites were the soft tissues in the region of the chest wall, axilla and infraclavicular fossa (Figures 5-10). One patient had a large chest wall mass with large axillary nodes, a very atypical finding (Figures 11).

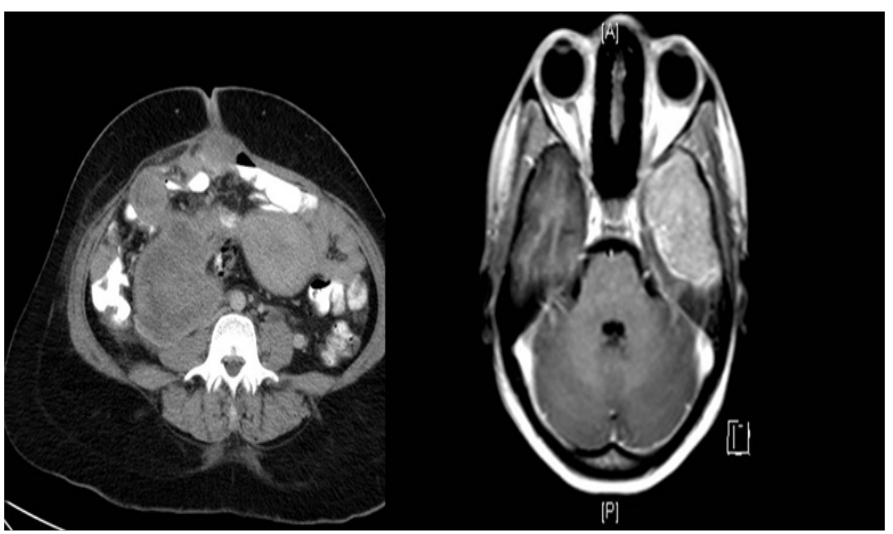

Figure I Contrast enhanced axial CT of the abdomen showing a pelvic and iliac fossa mass displacing the displacing the uterus with mesenteric and omental deposits.
Table I Location of the tumours was predominantly in the chest wall, limbs and extra skeletal sites

\begin{tabular}{|c|c|}
\hline Site of tumour & Number of patients \\
\hline Acetabulum & 2 \\
\hline Axilla & 1 \\
\hline Brain & I \\
\hline Chest wall & 7 \\
\hline Clavicle & 2 \\
\hline Femur & I \\
\hline Fibula & I \\
\hline Foot & 1 \\
\hline groin & I \\
\hline Hand & I \\
\hline Hip and pelvis & 5 \\
\hline Humerus & 4 \\
\hline Infraclavicular/supraclavicular region & 2 \\
\hline Mandible & I \\
\hline Ovary & I \\
\hline Pancreas & I \\
\hline Prostate & I \\
\hline Rectum & I \\
\hline Rib & 4 \\
\hline Sacrum & 2 \\
\hline Scapula & 4 \\
\hline Scrotum & I \\
\hline Tibia & 5 \\
\hline
\end{tabular}

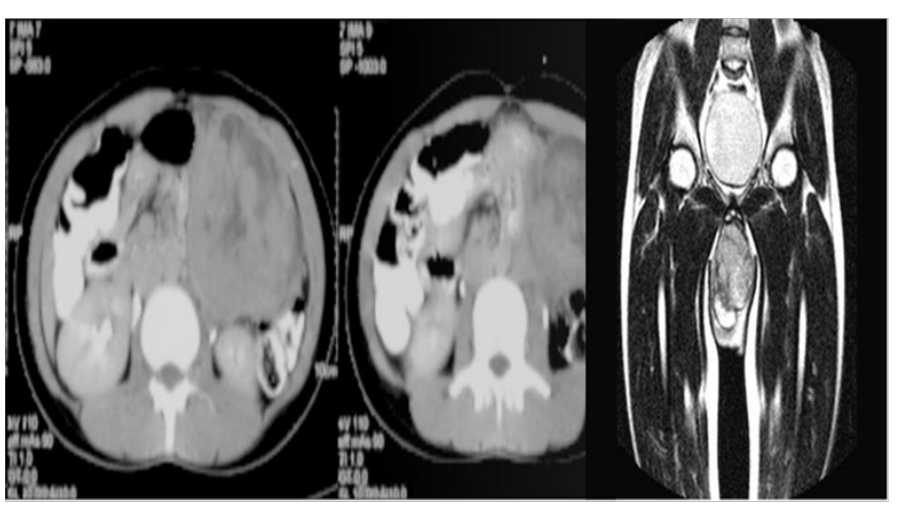

Figure 2 Contrast enhanced TI weighted images, MRI of the brain showing an intensely enhancing mass in the left middle cranial fossa.

Figure 3 Contrast enhanced CT axial sections of the abdomen showing a large heterogeneous mass arising from the pancreatic tail.

Figure 4T2 weighted coronal sections of the pelvis showing a heterogeneous mass in the scrotum. 


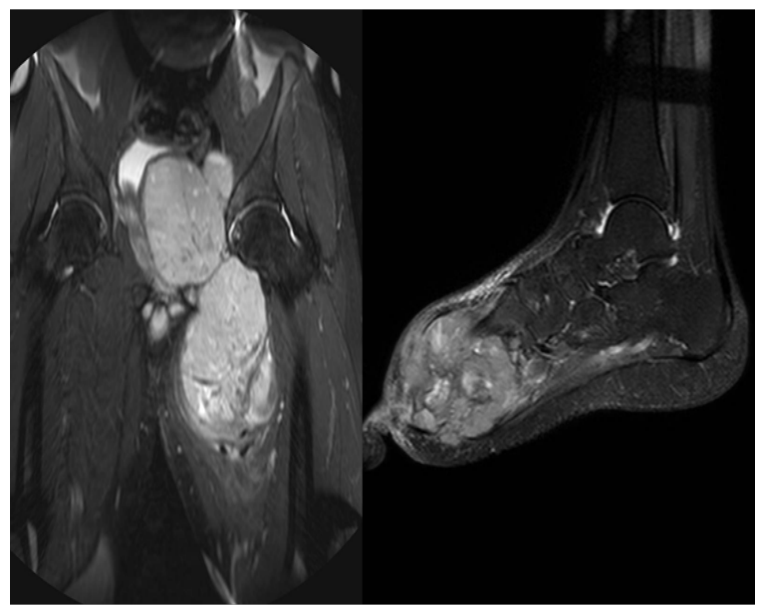

Figure 5 STIR coronal sequences of the pelvis, MRI images showing a heterogeneously hyperintense mass in the pelvis arising from the acetabulum.

Figure $6 \mathrm{MRI}$ of the foot, sagittal STIR sequence showing a heterogeneous mass involving the fore and mid foot.

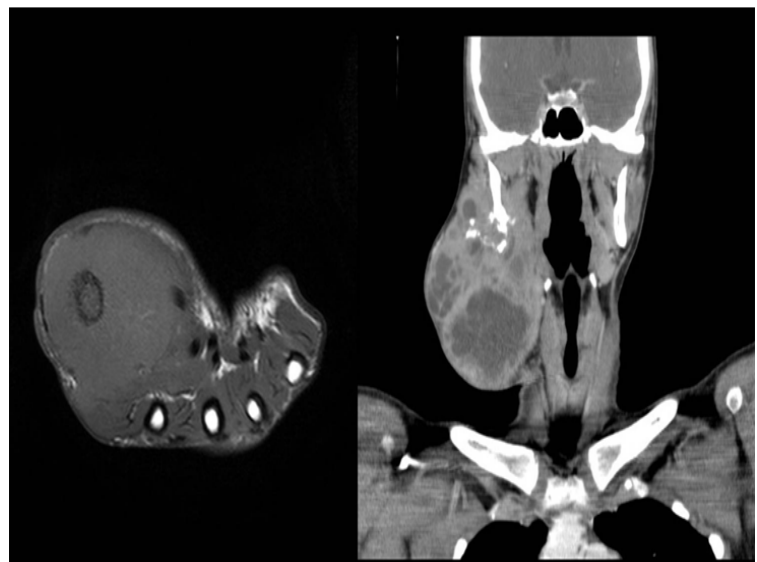

Figure $7 \mathrm{MRI}$ of the hand, STIR images showing a hypointense mass arising from the thumb.

Figure 8 Contrast enhanced, CT coronal sections showing a heterogeneous mass arising from the right mandible.

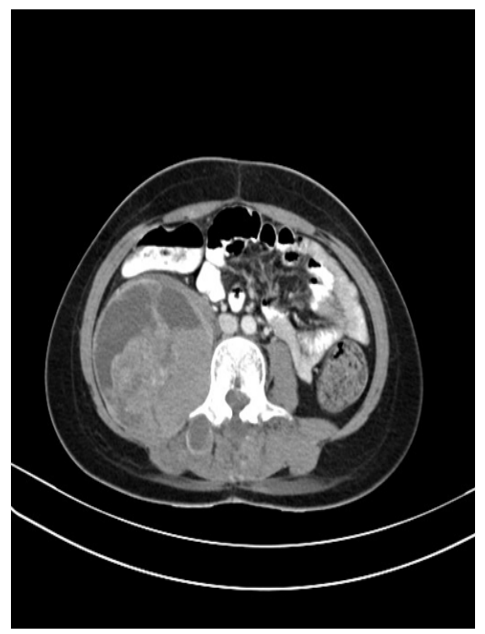

Figure 9 Contrast enhanced axial CT sections of abdomen showing a heterogeneous mass in the right para-spinal region.

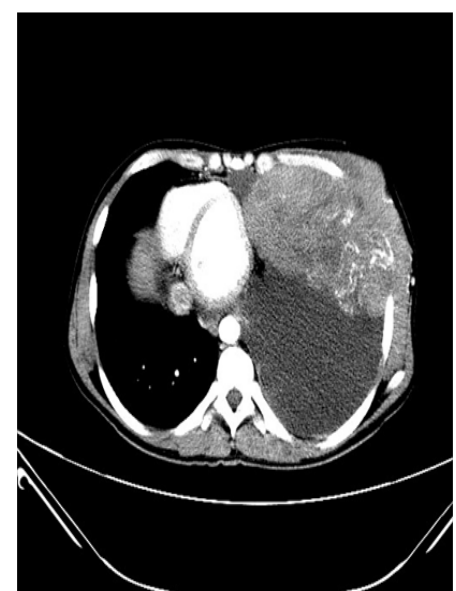

Figure 10 Contrast enhanced axial CT sections of the thorax showing a heterogenous mass arising from the chest wall.

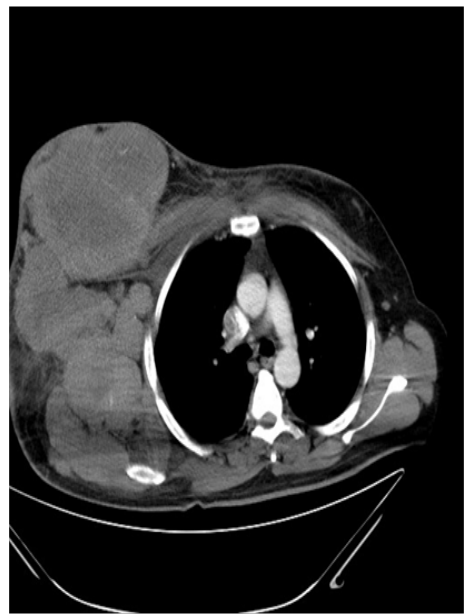

Figure I I Contrast enhanced axial CT sections of the thorax showing a heterogenous mass arising from the chest wall with large axillary lymph nodes.

\section{Discussion}

Due to the rare occurrence of EFT in adults, and with no published studies which have looked at the imaging findings exclusively in an adult population, this study has made an attempt to address this important issue. To the best of our knowledge, this article presents the largest series in the world of EFT in the adult age group. The incidence of $18 \%$ of extra skeletal EFT, is the highest reported so far as compared to $8 \%$ reported previously in a pediatric and adolescent population, ${ }^{5-8}$ This was a single centre study from a large tertiary care hospital in south India, which can be a subject of referral bias.

Concerning patient demographics, in our study, the mean age of presentation was about 23years. Male predominance is a feature in most previous studies but we showed a more than four is to one ratio in our population. The common sites included chest wall, illium, hip, shoulder, humerus and the bones forming the knee joint. Ewing sarcoma is vascular, and the extent of spread through the medullary cavity is markedly underestimated on radiographs. Therefore, MRI is highly recommended as the preferred imaging means for staging the tumor. MRI determines accurately the true extent of the lesion, as well as the involvement of critical adjacent structures including neurovascular involvement. 
EES is a rare small, undifferentiated, small, round cell tumor of uncertain origin. The compiled data including 1,505 patients of ESFT from the United States, Europe and Japan showed that EES cases represented $8 \%$ compared ES of bone which was about $87 \%$.In our series EES formed $14.9 \%$ of all tumours. It differs from skeletal ES in several respects. The average age of occurrence of EES is 20years, in comparison to 10years for skeletal ES. EES has a similar incidence in both genders, whereas skeletal ES has a reported 2:1 male predilection. ${ }^{5,6}$ Skeletal ES has a predilection for the long bones of the lower extremities. ${ }^{5}$ In contrast, EES has a varied anatomical distribution, commonly affecting the extremities (especially the lower extremities), soft tissues of the trunk, such as paravertebral and intercostal regions, pelvis, head and neck, and peritoneum. Other reported locations of EES include the mediastinum, heart, external genitalia, and broad ligament. ${ }^{6}$

The tumor tends to spread locally, infltrating deep fascial spaces and invading muscles or skeletal structures as well as other adjacent structures .The CT finding of EES is usually a heterogeneously enhancing mass often with areas of necrosis. ${ }^{7-9}$ On MR images, it is usually of low signal compared to muscle on T1-weighted images, of high signal intensity on T2-weighted images, and exhibits heterogeneous enhancement. ${ }^{10,11}$ Both local recurrences and distant metastases are common in EES. ${ }^{7}$ For bone tumours, a diagnosis of the bony component of ewing sarcoma is better evaluated on CT or plain films. Proper visualisation of small calcifications or fine periosteal reactions is of importance in predicting probable histological diagnosis. Calcification is shown as a signal void with MRI. Thus plain films remain the most reliable imaging method in the initial diagnosis of Ewing sarcoma. CT helps define bone destruction that is usually seen with Ewing sarcoma. Tumor size can be evaluated with contrast-enhanced CT scanning, which may be used in followup evaluation during chemotherapy. However, the preferred method in staging and following up Ewing sarcoma is still MRI.

MRI is essential to elucidate soft-tissue involvement because the tumor has low signal intensity on T1-weighted images compared with the normal high signal intensity of the bone marrow. On T2-weighted images, the tumor is hyperintense compared with muscle. Sequences with inversion recovery (STIR) sequence are also recommended. The accuracy of MRI-that is, the positive predictive value plus the negative predictive value-has been reported to be $90.3 \%$ in one study. ${ }^{12}$ In most patients, MRI seems to provide more specific information than CT scanning. Hence, MRI is the test of choice for staging in cases of suspected Ewing sarcoma.MR can also be used to monitor tumor response to chemotherapy. CT and MRI and often unable to differentiate among osteosarcomas, fibrosarcomas, and other malignant tumors, as well as osteomyelitis. However, Ewing sarcoma occurs in patients of a particular distinct age group, and its diagnosis is usually apparent.

Ewing sarcoma commonly infiltrates vessels and surrounding tissues. Local recurrence can occur frequently following radiotherapy, so it is very important to accurately determine the tumor extent before both surgery and radiotherapy. In tumors of the long bones, the soft tissue involvement is well seen with MRI and CT. ${ }^{13-16}$ The vascular relationships of the tumor are easily established with MRI even without contrast administration. ${ }^{13,15}$ When the tumor is small and conservative limb salvage surgery is planned, it is important for the surgeon to know whether the vessels are involved or not. Ewing sarcoma is often a diaphyseal tumor; thus it is important to assess accurately the extension in the region of the epiphyseal plate. For this purpose, MRI is much better than CT in defining the bone marrow extent. ${ }^{11}$ On the available radiographs, 25 of our patients had a visible periosteal reaction. Most of our patients had tumour localized to the diaphysis or metadiaphysis. The presence of haemorrhage was seen only in 2 tumors. All tumors except one had large soft tissue masses.

Bone cortex lacks mobile protons and thus has dark signal on all pulse sequences. Tumor invasion of bone cortex can be identified because of its prolonged T1 and T2 relaxation times. Although Zimmer et al. ${ }^{17}$ believed that cortical disease was more obviously shown on longer $\mathrm{SE}$ sequences ( $\mathrm{TE}=60 \mathrm{msec}, \mathrm{TR}=2,000 \mathrm{msec}$ ), in 8 cases with evident cortical disease on MR this was seen as well on T1as on T2-weighted MR pulse sequences. Periosteal reaction could be identified in 6 cases by Pettersson et al. ${ }^{18}$ has also commented on the advantage of MR in providing information on tumor relationship to major vessels. In general, T1-weighted images provided the greatest contrast between tumor and marrow, T2-weighted images showed the contrast between tumor and muscles, and tumor-infiltrating-bone cortex was seen with either, as reported previously. ${ }^{19,20}$

Vertebral manifestations of Ewing sarcoma may mimic those of hemangiomas. Other malignant tumors such as chondrosarcoma, fibrosarcoma, and soft-tissue sarcomas must be included in the differential diagnosis, although these usually occur in patients older than those with Ewing sarcoma. Owing to the use of chemotherapy as the first step of treatment, the prognosis for Ewing sarcoma has significantly improved. The prognosis of the loco regional disease has improved too, but conservative surgery is difficult in Ewing tumor because the patients are young, and the lesion is frequently central in location. In the long-term follow-up $((\mathrm{n}=28)$, most patients without recurrence one year after therapy showed a low signal in the surgical area in $\mathrm{T} 2$ weighted images.

\section{Conclusion}

The imaging characteristics in this largest case series of adult EFT reported so far were similar to those reported in the paediatric EFT. Our incidence of extra skeletal EFT of $18 \%$ is the highest reported so far in the adult population, this however needs to be confirmed in other series. MRI is the best single examination for preoperative evaluation of the extent of neoplastic involvement and follow-up of patients with EFT.

\section{Acknowledgements}

None.

\section{Conflict of interest}

Author declares that there is no conflict of interest.

\section{References}

1. Serhat Avcu, Hüseyin Akdeniz, Aydın Bora, et al. Primary Extraskeletal Ewing Sarcoma Originating from Chest Wall in a Child. Eur J Gen Med. 2010;7(1):101-103.

2. Henk CB, Grampp S, Weisbauer P, et al. Ewing sarcoma. Diagnostic Imaging. Radiologe. 1998;38(6):509-522.

3. Kerst JM, Van Coevorden F, Peterse J, et al. Young adults with Ewing's sarcoma. Ned Tijdschr Geneeskd. 2004;148(27):1355-1358.

4. Widhe B, Widhe TJ. Initial symptoms and clinical features in osteosarcoma and Ewing sarcoma. J Bone Joint Surg Am. 2000;82(5):667-674. 
5. Stuart Harris R, Wills EJ, Philips J, et al. Extraskeletal Ewing's sarcoma: a clinical, morphological and ultrastructural analysis of fve cases with a review of the literature. Eur J Cancer Clin Oncol. 1986;22(4):393-400.

6. Higgins JC, Katzman PJ, Yeager SB, et al. Extraskeletal Ewing's sarcoma of primary cardiac origin. Pediatr Cardiol. 1994;15(4):207-208.

7. Ahmad R, Mayol BR, Davis M, et al. Extraskeletal Ewing's sarcoma. Cancer. 1999;85:725-731.

8. Chang SK, Wang TL, Teh M. Extraskeletal Ewing's sarcoma presenting with pulmonary embolism. Australas Radiol. 1996;40(2):175-178.

9. Balakrishnan R, Khairullah QT, Giraldo A, et al. Extraskeletal Ewing's sarcoma in a kidney transplant patient. Am J Kidney Dis. 1999;33(6):1164-1167.

10. Lim TC, Tan WT, Lee YS. Congenital extraskeletal Ewing's sarcoma of the face: a case report. Head Neck. 1994;16(1):75-78.

11. Guyot-Drouot MH, Cotton A, Flipo RM, et al. Contribution of magnetic resonance imaging to the diagnosis of extraskeletal Ewing sarcoma. Rev Rhum Engl ed. 1999;66(10):516-519.

12. Hudson TM, Schakel M, Springfield DS. Limitation of CT following excisional biopsy of soft tissue sarcomas. Skeletal Radiol. 1985;13(1):49-54.
13. Pettersson H, Hamlin DJ, Mancuso A, et al. Magnetic resonance of the musculoskeletal system. Acta Radiol. 1985;26:225.

14. Smith J, Heelan RT, Huvos AG, et al. Radiographic changes in primary osteogenic sarcoma following intensive chemotherapy. Radiology. 1982;143(2):355-360.

15. Vanel D, Contesso G, Couanet D, et al. Computed tomography in evaluation of 41 cases of Ewing's sarcoma. Skeletal Radiol. 1982;9(1):8-13.

16. Richardson ML, Kilcoyne RF, Gillespy T, et al. Magnetic resonance imaging of musculoskeletal neoplasms. Radiol Clin North Am. 1986;24(2):259-267.

17. Zimrner WD, Berquist TH, McLeod RA, et al. Bone tumors: magnetic resonance imaging versus computed tomography. Radiology. 1985;155(3):709-718.

18. Pettersson H, Hamlin DJ, Mancuso A, et al. Magnetic resonance imaging of the musculoskeletal system. Acta Radiologica. 1985;26(3):225-234.

19. Christophe Frouge, Daniel Vanel, Christine Coffre, et al. The role of magnetic of Ewing sarcoma A report of 27 cases resonance imaging in the evaluation. Skeletal Radiology. 1988;17(6):387-392.

20. Orest Boyko, David A Cory, Cohen MD, et al. MR Imaging of osteosarcoma and Ewings Sarcoma. AJR Am J Roentgenol. 1987;148(2):317-322. 